\title{
SIMPLIFIED TIKHONOV REGULARIZATION FOR TWO KINDS OF PARABOLIC EQUATIONS
}

\author{
Li Jing AND WANG FANG
}

\begin{abstract}
This paper is devoted to simplified Tikhonov regularization for two kinds of parabolic equations, i.e., a sideways parabolic equation, and a two-dimensional inverse heat conduction problem. The measured data are assumed to be known approximately. We concentrate on the convergence rates of the simplified Tikhonov approximation of $u(x, t)$ and its derivative $u_{x}(x, t)$ of sideways parabolic equations at $0 \leq x<1$, and that of two-dimensional inverse heat conduction problem at $0<x \leq 1$, respectively.
\end{abstract}

\section{Introduction}

In several engineering contexts, there is sometimes a need to determine the temperature on both sides of a thick wall, but one side is inaccessible to measure (see e.g. [7]). In one-dimensional setting, this problem occasionally leads to the following parabolic equation in the quarter plane:

$$
\begin{aligned}
u_{t} & =a(x) u_{x x}+b(x) u_{x}+c(x) u, x>0, t>0, \\
u(1, t) & =g(t), t>0, \\
u(x, 0) & =0, x>0,
\end{aligned}
$$

where $a, b$ and $c$ are given functions such that there exist $\lambda, \Lambda>0$,

$$
\lambda \leq a(x) \leq \Lambda, c(x) \leq 0, x \in \mathbb{R}^{+} .
$$

For simplicity, we suppose that

$$
a(x) \in C^{2}\left(\mathbb{R}^{+}\right), b(x) \in C^{1}\left(\mathbb{R}^{+}\right), c(x) \in C\left(\mathbb{R}^{+}\right) .
$$

We want to know $u(x, t)$ for $0 \leq x<1$. This is a severely ill-posed problem (cf. $[2,4])$, i.e., if small changes of $g(t)$ with

$$
\left\|g-g_{\delta}\right\| \leq \delta
$$

Received October 4, 2009; Revised December 21, 2009.

2010 Mathematics Subject Classification. 35K05, 47A52, 65J10, 65N21.

Key words and phrases. Fourier transformation, simplified Tikhonov regularization, convergence rate, sideways parabolic equations, inverse heat conduction problems.

This work was partially supported by NNSF of China Grant No.10671211 and Hunan Provincial Natural Science Foundation of China Grant No. 07JJ3005. 
there may be dramatically large errors in the solution $u(x, t)$.

To solve this difficulty, the most popular method is regularization $[3,9$, 10, 17], i.e., using the solutions of well-posed problems to approximate the solutions of the original problems. A lot of authors deal with the above illposed problems by different methods. Roughly speaking, the methods involved are simplified Tikhonov regularization [4], Fourier method [2, 4], Wavelet Mayer method $[2,15]$, numerical method $[1,5,13,16,8]$.

In [4], using simplified Tikhonov regularization, Fu discussed the convergence rate of the approximate solution to $u(x, t)$ at $0<x<1$ with $g(t)$ replaced by $g_{\delta}(t)$. Under the condition that $\|f\|=\|u(0, t)\| \leq E$, the convergence rate of the approximate solution of the sideways parabolic equation may attain $O\left(\delta^{x}\right), 0<x<1$. However, he did not use this method to investigate the convergence rate at the boundary $x=0$. The present work is aimed to study the convergence rate of the approximation of $u(x, t)$ and $u_{x}(x, t)$ for all $0 \leq$ $x<1$. In addition, for two-dimensional inverse heat conduction problems (IHCP), suppose the data at $x=0$ are observed, we also need to get the information for $0<x \leq 1$. Up to now, many authors put forward methods on this topic, we can refer to $[11,14,18]$. However, few authors have investigated the convergence rate. Here, by simplified Tikhonov regularization, we can obtain the convergence rate of the approximate solution exactly.

The structure of this paper is as follows. In Section 2, we present some definitions and theorems that we need for our results and give the convergence rates of approximation of $u(x, t)$ and $u_{x}(x, t)$ for a sideways parabolic equation at $0 \leq x<1$. The convergence rate of approximate solution of the twodimensional inverse heat conduction problem for $0<x \leq 1$ is outlined in Section 3 when the available data are inaccurate. Our results improve and generalize many known results in this field [4].

\section{Convergence rate for sideways parabolic equations}

For brevity, the notation $\|\cdot\|$ denotes $L^{2}$-norm, and

$$
\hat{h}(\xi)=\frac{1}{\sqrt{2 \pi}} \int_{-\infty}^{+\infty} e^{-i \xi t} h(t) d t
$$

is the Fourier transform of function $h(t)$, then

$$
h(t)=\frac{1}{\sqrt{2 \pi}} \int_{-\infty}^{+\infty} e^{i \xi t} \hat{h}(\xi) d \xi .
$$

As we consider the problem in $L^{2}(\mathbb{R})$ with respect to variable $t$, we extend $u(x, \cdot), g(\cdot)=u(1, \cdot), f(\cdot)=u(0, \cdot)$ and other functions appearing in the section to be zero for $t<0$. To obtain our convergence rate, we need the following assumptions and lemmas first. We assume that there exists a priori bound for the function $f(t)=u(0, t)$ :

$$
\|f\| \leq E
$$


or

$$
\|f\|_{p} \leq E, p \geq 0
$$

where $\|f\|_{p}$ is defined by

$$
\|f\|_{p} \triangleq\left(\int_{-\infty}^{+\infty}\left(1+\xi^{2}\right)^{p}|\hat{f}(\xi)|^{2} d \xi\right)^{\frac{1}{2}} .
$$

Lemma $1([7])$. Let $v(x, \xi)$ be the solution of the following boundary value problem for ordinary differential equation

$$
\begin{aligned}
i \xi v(x, \xi) & =a(x) v_{x x}+b(x) v_{x}+c(x) v, x>0, \xi \in \mathbb{R}, \\
v(0, \xi) & =1, \\
\lim _{x \rightarrow \infty} v(x, \xi) & =0, \xi \neq 0
\end{aligned}
$$

for $\xi=0$ we require $v(x, 0)$ be bounded as $x$ tends to $\infty$. Suppose that the direct problem

$$
\left\{\begin{aligned}
u_{t} & =a(x) u_{x x}+b(x) u_{x}+c(x) u \\
u(x, 0) & =0 \\
u(0, t) & =f(t)
\end{aligned}\right.
$$

has a solution $u(x, t)$. Then

$$
u(x, t)=\frac{1}{\sqrt{2 \pi}} \int_{-\infty}^{+\infty} e^{i \xi t} v(x, \xi) \hat{f}(\xi) d \xi, x>0
$$

and

$$
\hat{u}(x, \xi)=v(x, \xi) \hat{f}(\xi) .
$$

Remark 1. From Lemma 1 , it is obvious that if $u$ is the solution of the above direct problem, then $\hat{u}(1, \xi)=v(1, \xi) \hat{f}(\xi)=\hat{g}(\xi)$ and $\hat{g}(\xi)=\frac{v(1, \xi)}{v(x, \xi)} \hat{u}(x, \xi)$. So,

$$
\hat{u}_{x}(x, \xi)=v_{x}(x, \xi) \hat{f}(\xi)=\frac{v_{x}(x, \xi)}{v(1, \xi)} \hat{g}(\xi) .
$$

Lemma $2([7])$. There exist constants $c_{k}, k=1,2,3,4$, such that for $x \in[0,1]$ and $|\xi|$ large enough, say $|\xi| \geq \bar{\xi}$

$$
\begin{gathered}
c_{1} e^{-A(x) \sqrt{|\xi| / 2}} \leq|v(x, \xi)| \leq c_{2} e^{-A(x) \sqrt{|\xi| / 2}} \\
c_{3} \sqrt{|\xi|} e^{-A(x) \sqrt{|\xi| / 2}} \leq\left|v_{x}(x, \xi)\right| \leq c_{4} \sqrt{|\xi|} e^{-A(x) \sqrt{|\xi| / 2}}
\end{gathered}
$$

where $A(x)=\int_{0}^{x} \frac{1}{\sqrt{a(s)}} d s$. Especially, the right-hand side inequalities in (8) and (9) are valid for all $\xi \in \mathbb{R}$ with other constants $c_{2}^{\prime}$ and $c_{4}^{\prime}$.

Lemma 3 ([7]). If the boundary value problem

$$
a(x) v_{x x}(x)+b(x) v_{x}(x)+c(x) v(x)=0,0<x<\infty ;
$$




$$
v(0)=1, v(x) \text { is bounded as } x \rightarrow \infty
$$

has a unique solution, then there exist constants $c_{1}^{\prime}$ and $c_{2}^{\prime}$ such that

$$
c_{1}^{\prime} e^{-A(1) \sqrt{|\xi| / 2}} \leq|v(1, \xi)| \leq c_{2}^{\prime} e^{-A(1) \sqrt{|\xi| / 2}}, \forall \xi \in \mathbb{R} .
$$

Refer to [4], for all $0 \leq x<1$, we can define an operator $K(x): L^{2}(\mathbb{R}) \rightarrow$ $L^{2}(\mathbb{R})$ by

$$
\widehat{K(x) h}(x, \xi)=\frac{v(1, \xi)}{v(x, \xi)} \hat{h}(x, \xi) .
$$

Let $u(x, t)$ be the solution of (1)-(3). Then (1)-(3) can be rewritten as

$$
K(x) u(x, t)=g(t), 0 \leq x<1 .
$$

Since (1)-(3) is ill-posed, as we all know, to solve this difficulty, we often use the solution of Tikhonov function

$$
\min \left\{\left\|K(x) h-g_{\delta}\right\|^{2}+\alpha\|h\|^{2}\right\}
$$

over all $h(x, \cdot)$ in $L^{2}(\mathbb{R})$ to approximate to $u(x, t)$ for $0 \leq x<1$, where $\alpha>0$ is the regular parameter.

By mathematical analysis, we can easily get the following results.

Lemma 4. For $0 \leq x \leq 1, \alpha>0$, there hold the inequalities:

(1) $\sup _{s \geq 0} \frac{e^{(1-x) s}}{1+\alpha e^{2 s}} \leq \alpha^{(x-1) / 2}, \sup _{s \geq 0} \frac{e^{x s}}{1+\alpha e^{2 s}} \leq \alpha^{-x / 2}$;

(2) $\sup _{s \geq 0} \frac{e^{(2-x) s}}{1+\alpha e^{2 s}} \leq \alpha^{(x-2) / 2}, \sup _{s \geq 0} \frac{e^{(1+x) s}}{1+\alpha e^{2 s}} \leq \alpha^{-(1+x) / 2}$.

Definition 1 (cf. [4]). Let

$$
u_{\delta}(x, t) \triangleq \frac{1}{\sqrt{2 \pi}} \int_{-\infty}^{+\infty} e^{i \xi t} \frac{(v(x, \xi) / v(1, \xi)) \hat{g}_{\delta}(\xi)}{1+\alpha|1 / v(1, \xi)|^{2}} d \xi
$$

and it is called a simplified Tikhonov approximate solution of $u(x, t)$ of problem (1)-(3). Then $\hat{u}_{\delta x}=\frac{\left(v_{x}(x, \xi) / v(1, \xi)\right) \hat{g}_{\delta}(\xi)}{1+\alpha|1 / v(1, \xi)|^{2}}$.

In [4], for $0<x<1, \mathrm{Fu}$ investigated the convergence rate of the above simplified Tikhonov approximate solution with $\alpha=\left(\frac{\delta}{E}\right)^{2}$ under the condition that (5) holds,

$$
\left\|u_{\delta}(x, \cdot)-u(x, \cdot)\right\| \leq C \delta^{\frac{A(x)}{A(1)}} E^{1-\frac{A(x)}{A(1)}} .
$$

We are now in the position to formulate the convergence rate for all $0 \leq x<$ 1.

Theorem 1. Suppose problem (1)-(3) has a unique solution, let $u(x, t)$ given by $(7)$ be the exact data at $0 \leq x<1$, and $u_{\delta}(x, t)$ be the simplified Tikhonov regularized approximation to $u(x, t)$. In addition, assume that the measured history (noisy data) $g_{\delta}(t)$ satisfy (4) and priori condition (6) be valid, then if $\alpha=\left(\frac{\delta}{E}\right)^{\gamma}, 1 \leq \gamma \leq 2$, there holds

(14) $\left\|u_{\delta}(x, \cdot)-u(x, \cdot)\right\|=O\left(\delta^{1-\gamma / 2} \cdot \delta^{\frac{\gamma A(x)}{2 A(1)}}\right)+O\left(\delta^{\gamma-1} \cdot \delta^{\frac{A(x)}{2 A(1)}}\left(\ln \frac{E}{\delta}\right)^{-p+\frac{A(x) p}{2 A(1)}}\right)$ 


$$
+O\left(\delta^{\frac{\gamma A(x)}{2 A(1)}}\left(\ln \frac{E}{\delta}\right)^{-2 p}\right), p \geq 0,0 \leq x<1 .
$$

Proof. The same argument as Theorem 2.4 in [4], we can obtain

$$
\begin{aligned}
\left\|\hat{u}(x, \cdot)-\hat{u}_{\delta}(x, \cdot)\right\|^{2} & \leq 2 \int_{-\infty}^{+\infty}\left|\hat{g}(\xi)-\hat{g}_{\delta}(\xi)\right|^{2} I_{1} d \xi+2 \alpha^{2} \int_{-\infty}^{+\infty}|\hat{f}(\xi)|^{2} I_{2} d \xi \\
& \triangleq J_{1}+J_{2},
\end{aligned}
$$

where with $2 s=\sqrt{2|\xi|}, C_{1}, C_{2}>0$,

$$
I_{1}=\frac{|v(x, \xi) / v(1, \xi)|^{2}}{\left(1+\alpha|1 / v(1, \xi)|^{2}\right)^{2}} \leq C_{1}\left(\frac{e^{(A(1)-A(x)) s}}{1+\alpha e^{2 A(1) s}}\right)^{2} \leq C_{1} \alpha^{(A(x)-A(1)) / A(1)}
$$

and

$$
I_{2}=\frac{|1 / v(1, \xi)|^{2} \cdot|v(x, \xi) / v(1, \xi)|^{2}}{\left(1+\alpha|1 / v(1, \xi)|^{2}\right)^{2}} \leq C_{2}\left(\frac{e^{(2 A(1)-A(x)) s}}{1+\alpha e^{2 A(1) s}}\right)^{2}
$$

Thus, $J_{1} \leq 2 \delta^{2} \sup _{\xi \in \mathbb{R}} I_{1} \leq 2 C_{1} \delta^{2} \alpha^{(A(x)-A(1)) / A(1)}=2 C_{1} \frac{\delta^{2}}{\alpha} \cdot \alpha^{\frac{A(x)}{A(1)}}$.

Next, it remains to give the estimation of $J_{2}$.

Setting $\xi_{0}=2\left[\frac{1}{2 A(1)} \ln \left(\frac{E}{\delta}\left(\ln \frac{E}{\delta}\right)^{-p}\right)\right]^{2}$, by Lemma 4, we come to the relation

$$
\begin{aligned}
J_{2} \leq & 2 C_{2} \alpha^{2} \int_{-\infty}^{+\infty}|\hat{f}(\xi)|^{2}\left(\frac{e^{(2 A(1)-A(x)) s}}{1+\alpha e^{2 A(1) s}}\right)^{2} d \xi \\
= & 2 C_{2} \alpha^{2} \int_{|\xi| \leq \xi_{0}}|\hat{f}(\xi)|^{2}\left(1+\xi^{2}\right)^{p}\left[\frac{e^{(2 A(1)-A(x)) s}}{\left(1+\alpha e^{2 A(1) s}\right)\left(1+\xi^{2}\right)^{\frac{p}{2}}}\right]^{2} d \xi \\
& +2 C_{2} \alpha^{2} \int_{|\xi| \geq \xi_{0}}|\hat{f}(\xi)|^{2}\left(1+\xi^{2}\right)^{p}\left[\frac{e^{(2 A(1)-A(x)) s}}{\left(1+\alpha e^{2 A(1) s}\right)\left(1+\xi^{2}\right)^{\frac{p}{2}}}\right]^{2} d \xi \\
\leq & 2 C_{2} \alpha^{2} E^{2}\left(\left.e^{(2 A(1)-A(x)) s_{0}}\right|_{\left.s_{0}=\sqrt{\xi_{0} / 2}\right)^{2}+2 C_{2} \alpha^{2} E^{2} \alpha^{A(x) / A(1)-2} \xi_{0}^{-2 p}}\right. \\
= & 2 C_{2} E^{2} \alpha^{2}\left(\frac{E}{\delta}\right)^{2-\frac{A(x)}{A(1)}}\left(\ln \frac{E}{\delta}\right)^{-2 p+\frac{A(x) p}{A(1)}}+2 C_{2} E^{2} \xi_{0}^{-2 p} \alpha^{A(x) / A(1)} \\
= & 2 C_{2} E^{4-\frac{A(x)}{A(1)}}\left(\frac{\alpha}{\delta}\right)^{2} \delta^{\frac{A(x)}{A(1)}}\left(\ln \frac{E}{\delta}\right)^{-2 p+\frac{A(x) p}{A(1)}}+2 C_{2} E^{2} \xi_{0}^{-2 p} \alpha^{A(x) / A(1)} .
\end{aligned}
$$

Consequently, with the help of Parseval equality,

$$
\begin{aligned}
& \left\|u(x, \cdot)-u_{\delta}(x, \cdot)\right\|^{2} \\
= & \left\|\hat{u}(x, \cdot)-\hat{u}_{\delta}(x, \cdot)\right\|^{2} \\
\leq & 2 C_{1} \frac{\delta^{2}}{\alpha} \cdot \alpha^{\frac{A(x)}{A(1)}}+2 C_{2} E^{4-\frac{A(x)}{A(1)}}\left(\frac{\alpha}{\delta}\right)^{2} \delta^{\frac{A(x)}{A(1)}}\left(\ln \frac{E}{\delta}\right)^{-2 p+\frac{A(x) p}{A(1)}} \\
& +2 C_{2} E^{2} \xi_{0}^{-2 p} \alpha^{A(x) / A(1)} \\
= & O\left(\delta^{2-\gamma} \cdot \delta^{\gamma A(x) / A(1)}\right)+O\left(\delta^{2(\gamma-1)} \delta^{A(x) / A(1)}\left(\ln \frac{E}{\delta}\right)^{-2 p+\frac{A(x) p}{A(1)}}\right)
\end{aligned}
$$




$$
+O\left(\delta^{\gamma A(x) / A(1)}\left(\ln \frac{E}{\delta}\right)^{-4 p}\right) .
$$

Hence the assertion follows.

Remark 2. From the discussion above, it follows that if $\gamma=2, p \geq 0$,

$$
\left\|u(x, \cdot)-u_{\delta}(x, \cdot)\right\| \leq C \delta^{\frac{A(x)}{A(1)}}, 0<x<1,
$$

which is the optimal order (see [4]).

Remark 3. Especially, to get the convergence rate of the approximate solutions at $x=0$, we only need to fix $p>0,1 \leq \gamma<2$.

In heat conduction applications, $u(x, t)$ in (1)-(3) denotes the temperature, and $u_{x}(x, t)$ denotes its flux, thus it is also valuable to estimate the approximation of $u_{x}(x, t)$.

Theorem 2. Suppose problem (1)-(3) has a unique solution, let $u(x, t)$ given by $(7)$ be the exact data at $0 \leq x<1$, and $u_{\delta}(x, t)$ be the simplified Tikhonov regularized approximation to $u(x, t)$. In addition, assume that the measured history (noisy data) $g_{\delta}(t)$ satisfy (4) and priori condition (6) with $p \geq 1 / 2$ be valid, then if $\alpha=\frac{\delta}{E}$, there holds

(17) $\left\|u_{\delta x}(x, \cdot)-u_{x}(x, \cdot)\right\|=O\left(\delta^{\frac{1}{4}}\right)+O\left(\delta^{\frac{A(x)}{2 A(1)}}\left[\left(\ln \frac{E}{\delta}\right)^{-p+\frac{A(x) p}{2 A(1)}}+\left(\ln \frac{E}{\delta}\right)^{1-2 p}\right]\right)$.

Proof. Along the line of the proof of Theorem 2.4 in [4], we can obtain

$$
\begin{aligned}
\left|\hat{u}_{x}(x, \cdot)-\hat{u}_{\delta x}(x, \cdot)\right|^{2} & \leq 2 \frac{\left|\frac{v_{x}(x, \xi)}{v(1, \xi)}\right|^{2} \cdot\left|\hat{g_{\delta}}-\hat{g}\right|^{2}}{\left[1+\alpha\left|\frac{1}{v(1, \xi)}\right|^{2}\right]^{2}}+2 \alpha^{2} \frac{\left|\frac{1}{v(1, \xi)}\right|^{2} \cdot\left|\frac{v_{x}(x, \xi)}{v(1, \xi)}\right|^{2} \cdot|\hat{f}|^{2}}{\left[1+\alpha\left|\frac{1}{v(1, \xi)}\right|^{2}\right]^{2}} \\
& =2\left|\hat{g_{\delta}}-\hat{g}\right|^{2} I_{1}+2 \alpha^{2}|\hat{f}|^{2} I_{2} .
\end{aligned}
$$

By Lemma 2 and Lemma 3, with $2 s=\sqrt{2|\xi|}$, one has

$$
\begin{aligned}
I_{1}=\frac{\left|\frac{v_{x}(x, \xi)}{v(1, \xi)}\right|^{2}}{\left[1+\alpha\left|\frac{1}{v(1, \xi)}\right|^{2}\right]^{2}} & \leq 2 \frac{\left(\frac{c_{4}}{c_{1}^{\prime}}\right)^{2}|\xi| e^{(A(1)-A(x)) \sqrt{2|\xi|}}}{\left[1+\left(\frac{1}{c_{2}^{\prime}}\right)^{2} \alpha e^{A(1) \sqrt{2|\xi|}]^{2}}\right.} \\
& \leq 2 \frac{\left(\frac{c_{4}}{c_{1}^{\prime}}\right)^{2}|\xi| e^{(A(1)-A(x)) \sqrt{2|\xi|}}}{\min \left\{1,\left(\frac{1}{c_{2}^{\prime}}\right)^{2}\right\}\left[1+\alpha e^{A(1) \sqrt{2|\xi|}}\right]^{2}} \\
& \leq C_{1}\left[\frac{s e^{(A(1)-A(x)) s}}{1+\alpha e^{2 A(1) s}}\right]^{2} .
\end{aligned}
$$

Similarly

$$
I_{2}=\frac{\left|\frac{1}{v(1, \xi)}\right|^{2} \cdot\left|\frac{v_{x}(x, \xi)}{v(1, \xi)}\right|^{2}}{\left[1+\alpha\left|\frac{1}{v(1, \xi)}\right|^{2}\right]^{2}} \leq C_{2}\left[\frac{s e^{(2 A(1)-A(x)) s}}{1+\alpha e^{2 A(1) s}}\right]^{2} .
$$




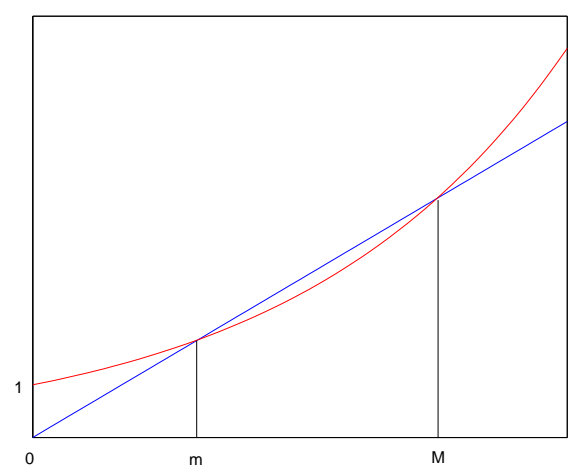

Figure 1

Define $p(s) \triangleq e^{\frac{A(1)}{2} s}-s$. Then $p_{\min }=\left.p\left(s_{0}\right)\right|_{s_{0}=\frac{2}{A(1)} \ln \frac{2}{A(1)}}=\frac{2}{A(1)}\left(1-\ln \frac{2}{A(1)}\right)$. Thereby, if $\frac{2}{A(1)} \leq e$, then $p_{\min } \geq 0$ and $s \leq e^{\frac{A(1)}{2} s}, \forall s \in \mathbb{R}^{+}$; Else if $\frac{2}{A(1)}>e$, from Figure 1, we know that there exist $m=m(A(1)), M=M(A(1))$ such that if $s \leq m$ or $s \geq M, s \leq e^{\frac{A(1)}{2} s}$, and if $m<s<M, s \geq e^{\frac{A(1)}{2} s}$. Therefore, according to Lemma 4 ,

$$
\begin{aligned}
I_{1} & \leq C_{1}\left[\frac{s e^{(A(1)-A(x)) s}}{1+\alpha e^{2 A(1) s}}\right]^{2} \\
& \leq C_{1} \max \left\{\left[\frac{M e^{(A(1)-A(x)) s}}{1+\alpha e^{2 A(1) s}}\right]^{2},\left[\frac{e^{3 A(1) s / 2}}{1+\alpha e^{2 A(1) s}}\right]^{2}\right\} \\
& \leq C_{1} \max \left\{M^{2} \alpha^{\frac{A(x)-A(1)}{A(1)}}, 3^{\frac{3}{2}} \alpha^{-3 / 2}\right\} .
\end{aligned}
$$

Remember that

$$
\left\|\hat{u}_{x}(x, \cdot)-\hat{u}_{\delta x}(x, \cdot)\right\|^{2} \leq 2 \int_{\mathbb{R}}\left|\hat{g}_{\delta}-\hat{g}\right|^{2} I_{1} d \xi+2 \alpha^{2} \int_{\mathbb{R}}|\hat{f}|^{2} I_{2} d \xi \triangleq J_{1}+J_{2} .
$$

Here,

(19) $J_{1}=2 \int_{\mathbb{R}}\left|\hat{g_{\delta}}-\hat{g}\right|^{2} I_{1} d \xi \leq 2 C_{1} \max \left\{M^{2} \alpha^{\frac{A(x)-A(1)}{A(1)}}, 3^{\frac{3}{2}} \alpha^{-3 / 2}\right\} \delta^{2}=O\left(\delta^{1 / 2}\right) ;$

$$
\begin{aligned}
J_{2} & =2 \alpha^{2} \int_{\mathbb{R}}|\hat{f}|^{2} I_{2} d \xi \\
& \leq 2 C_{2} \alpha^{2} \int_{\mathbb{R}}|\hat{f}|^{2}\left(1+\xi^{2}\right)^{p}\left[\frac{s e^{(2 A(1)-A(x)) s}}{\left(1+\alpha e^{2 A(1) s}\right)\left(1+4 s^{4}\right)^{p / 2}}\right]^{2}
\end{aligned}
$$


Note that if $0 \leq s \leq 1$, then $s \leq\left(1+4 s^{4}\right)^{p / 2}$, i.e., $\frac{s}{\left(1+4 s^{4}\right)^{p / 2}} \leq 1$. And if $s \geq 1$, we also have $\frac{s}{\left(1+4 s^{4}\right)^{p / 2}} \leq \frac{s}{\left(4 s^{4}\right)^{p / 2}}=2^{-p} s^{1-2 p} \leq 1$.

Consequently, setting $\xi_{0}=2\left[\frac{1}{2 A(1)} \ln \left(\frac{E}{\delta}\left(\ln \frac{E}{\delta}\right)^{-p}\right)\right]^{2}$, we get

$$
\begin{aligned}
J_{2} \leq & 2 C_{2} \alpha^{2} \int_{|\xi| \leq \xi_{0}}|\hat{f}|^{2}\left(1+\xi^{2}\right)^{p}\left[\frac{s e^{(2 A(1)-A(x)) s}}{\left(1+\alpha e^{2 A(1) s)\left(1+4 s^{4}\right)^{p / 2}}\right]^{2}}\right. \\
& +2 C_{2} \alpha^{2} \int_{|\xi| \geq \xi_{0}}|\hat{f}|^{2}\left(1+\xi^{2}\right)^{p}\left[\frac{s e^{(2 A(1)-A(x)) s}}{\left(1+\alpha e^{2 A(1) s}\right)\left(1+4 s^{4}\right) p / 2}\right]^{2} \\
\leq & 2 C_{2} E^{2} \alpha^{2} \sup _{|\xi| \leq \xi_{0}}\left[\frac{e^{(2 A(1)-A(x)) s}}{1+\alpha e^{2 A(1) s}}\right]^{2} \\
& +2 C_{2} E^{2} \alpha^{2} \sup _{|\xi| \geq \xi_{0}}\left[\frac{s e^{(2 A(1)-A(x)) s}}{\left(1+\alpha e^{2 A(1) s}\right)\left(1+4 s^{4}\right) p / 2}\right]^{2} \\
\leq & 2 C_{2} E^{2} \alpha^{2}\left(e^{(2 A(1)-A(x)) s_{0}} \mid s_{0}=\sqrt{\xi_{0} / 2}\right)^{2}+2 C_{2} E^{2} \alpha^{2} \alpha^{\frac{A(x)}{A(1)}-2} s_{0}^{2-4 p} \\
= & 2 C_{2} E^{4-\frac{A(x)}{A(1)}}\left(\frac{\alpha}{\delta}\right)^{2} \delta^{\frac{A(x)}{A(1)}}\left(\ln \frac{E}{\delta}\right)^{-2 p+\frac{A(x) p}{A(1)}}+2 C_{2} E^{2} s_{0}^{2-4 p} \alpha^{\frac{A(x)}{A(1)}} \\
= & O\left(\delta^{\frac{A(x)}{A(1)}}\left[\left(\ln \frac{E}{\delta}\right)^{-2 p+\frac{A(x) p}{A(1)}}+\left(\ln \frac{E}{\delta}\right)^{2-4 p}\right]\right) .
\end{aligned}
$$

Combing (18)-(20) with (21), we can get the assertion immediately.

\section{Convergence rate for two-dimensional inverse heat conduction problems}

In this section, we study a two-dimensional inverse heat conduction problem in a semi-infinite slab $[11,14]$, i.e.,

$$
\begin{aligned}
& u_{t}(x, y, t)=u_{x x}(x, y, t)+u_{y y}(x, y, t), 0<x<1, y>0, t>0, \\
& u(0, y, t)=\varphi(y, t), y>0, t>0
\end{aligned}
$$

with corresponding measured data function $\varphi_{\delta}(y, t)$,

$$
\begin{aligned}
u_{x}(0, y, t) & =0, y \geq 0, t \geq 0, \\
u(x, y, 0) & =0,0<x<1, y>0, \\
u(x, 0, t) & =0,0<x<1, t \geq 0 .
\end{aligned}
$$

Now, we wish to determine the temperature $u(x, y, t)$ for $0<x \leq 1$ from temperature $\varphi_{\delta}(y, t)$. In order to use some results from Fourier analysis, we extend the function $u(x, \cdot, \cdot), \varphi(\cdot, \cdot), \varphi_{\delta}(\cdot, \cdot)$ and $\psi(\cdot, \cdot)=u(1, \cdot, \cdot)$ to whole real $(y, t)$ plane by defining them to be zero everywhere in $\{(y, t), y<0, t<0\}$. We also assume that these functions are in $L^{2}\left(\mathbb{R}^{2}\right)$ and use the corresponding 
$L^{2}$ norm, as defined below

$$
\|\varphi\|=\left(\int_{\mathbb{R}^{2}}|\varphi(y, t)|^{2} d y d t\right)^{\frac{1}{2}} .
$$

We now could assume that the measured data function $\varphi_{\delta}(y, t)$ satisfies

$$
\left\|\varphi-\varphi_{\delta}\right\| \leq \delta
$$

where $\delta>0$ represents a bound on the measurement error.

Let

$$
\hat{\varphi}(\xi, \tau)=\frac{1}{2 \pi} \int_{\mathbb{R}^{2}} \varphi(y, t) e^{-i(\xi y+\tau t)} d y d t
$$

be the Fourier transform of a function $\varphi(y, t)$. Then

$$
\varphi(y, t)=\frac{1}{2 \pi} \int_{\mathbb{R}^{2}} \hat{\varphi}(\xi, \tau) e^{i(\xi y+\tau t)} d \xi d \tau .
$$

Taking Fourier transformation to (22), then

$$
\widehat{u}_{x x}(x, \xi, \tau)=\left(i \tau+\xi^{2}\right) \hat{u}(x, \xi, \tau) .
$$

Suppose $u$ is the solution of (22)-(26), we can easily get the solution by conditions (23) and (24) (one can refer to $[5,6,12]$ ), i.e.,

$$
\begin{aligned}
& \hat{u}(x, \xi, \tau)=\hat{\varphi}(\xi, \tau) \cosh (x \theta), 0 \leq x \leq 1, \xi, \tau \in \mathbb{R}, \\
& u(x, y, t)=\frac{1}{2 \pi} \int_{\mathbb{R}^{2}} \hat{\varphi}(\xi, \tau) \cosh (x \theta) e^{i(\xi y+\tau t)} d \xi d \tau,
\end{aligned}
$$

here $\theta$ is the principal value of $\sqrt{i \tau+\xi^{2}}$ :

$$
\theta=\sqrt{i \tau+\xi^{2}}=\sqrt{\frac{\sqrt{\tau^{2}+\xi^{4}}+\xi^{2}}{2}}+i \operatorname{sign}(\tau) \sqrt{\frac{\sqrt{\tau^{2}+\xi^{4}}-\xi^{2}}{2}} .
$$

Setting $a=\sqrt{\frac{\sqrt{\tau^{2}+\xi^{4}}+\xi^{2}}{2}}, b=\sqrt{\frac{\sqrt{\tau^{2}+\xi^{4}}-\xi^{2}}{2}}, \sigma=\operatorname{sign}(\tau)$. Then $\theta=a+i \sigma b$. It is obvious that

$$
\hat{\psi}(\xi, \tau)=\hat{u}(1, \xi, \tau)=\hat{\varphi}(\xi, \tau) \cosh \theta
$$

Since $|\cosh (x \theta)|$ is unbounded for $0<x \leq 1$, from (31) we can see that small errors in the data can blow up and completely destroy the solution for $0<x \leq 1$, thus the two-dimensional IHCP is ill-posed. Here, we use the simplified Tikhonov regularization to solve this difficulty.

Define $K(x): L^{2}\left(\mathbb{R}^{2}\right) \rightarrow L^{2}\left(\mathbb{R}^{2}\right)$ by

$$
\widehat{K(x) u}(x, \xi, \tau)=\frac{\hat{u}(x, \xi, \tau)}{\cosh (x \theta)} .
$$

Then (22)-(26) can be rewritten as

$$
K(x) u(x, y, t)=\varphi(y, t), 0 \leq x<1 .
$$


We use the solution of the following functional

$$
\min _{h(x, \cdot, \cdot) \in L^{2}\left(\mathbb{R}^{2}\right)} J(x), J(x)=\left\|K(x) h-\varphi_{\delta}\right\|^{2}+\alpha\|h\|^{2}
$$

to approximate to $u(x, y, t)$, where $\alpha>0$ is the regular parameter.

Lemma 5. There exists a unique solution to the above minimization problem (33). It is given by

$$
h_{\delta}(x, y, t)=\frac{1}{2 \pi} \int_{\mathbb{R}^{2}} e^{i(\xi y+\tau t)} \frac{\cosh (x \theta) \hat{\varphi}_{\delta}}{1+\alpha|\cosh (x \theta)|^{2}} d \xi d \tau .
$$

Proof. Let $I$ denote the identity operator in $L^{2}\left(\mathbb{R}^{2}\right)$ and $K^{*}(x)$ be the adjoint of $K(x)$. Then, the unique solution of the minimization problem (33) is given by

$$
h_{\delta}=\left[K^{*}(x) K(x)+\alpha I\right]^{-1} K^{*}(x) \varphi_{\delta} .
$$

By Parseval formula, one has

$$
\langle\widehat{K(x) u}, \hat{v}\rangle=\langle K(x) u, v\rangle=\left\langle u, K^{*}(x) v\right\rangle=\left\langle\hat{u}, \widehat{K(x)^{*} v} .\right.
$$

According to (32),

$$
\langle\widehat{K(x) u}, \hat{v}\rangle=\left\langle\frac{1}{\cosh (x \theta)} \hat{u}, \hat{v}\right\rangle=\left\langle\hat{u}, \frac{1}{\overline{\cosh (x \theta)}} \hat{v}\right\rangle .
$$

This immediately leads to,

$$
\widehat{K^{*}(x)} v=\frac{1}{\overline{\cosh (x \theta)}} \hat{v} .
$$

Consequently,

$$
K^{*} \widehat{(x) K(}(x) v=\frac{1}{|\cosh (x \theta)|^{2}} \hat{v}
$$

In view of

$$
K^{*} \widehat{(x) K(x)} h_{\delta}+\alpha \widehat{h_{\delta}}=\widehat{K^{*}(x) \varphi_{\delta}}
$$

we obtain

$$
\left(\frac{1}{|\cosh (x \theta)|^{2}}+\alpha\right) \hat{h}_{\delta}=\frac{1}{\overline{\cosh (x \theta)}} \hat{\varphi}_{\delta}
$$

It turns out that

$$
\begin{aligned}
\hat{h}_{\delta} & =\frac{\frac{1}{\overline{\cosh (x \theta)}} \hat{\varphi}_{\delta}}{\alpha+\frac{1}{|\cosh (x \theta)|^{2}}} \\
& =\frac{\cosh (x \theta) \hat{\varphi}_{\delta}}{1+\alpha|\cosh (x \theta)|^{2}} .
\end{aligned}
$$

Using the inverse Fourier transformation, we can get the assertion. 
Definition 2. Let

$$
u_{\delta}(x, y, t)=\frac{1}{2 \pi} \int_{\mathbb{R}^{2}} e^{i(\xi y+\tau t)} \frac{\cosh (x \theta) \hat{\varphi}_{\delta}}{1+\alpha|\cosh \theta|^{2}} d \xi d \tau
$$

and it is called a simplified Tikhonov approximate solution of $u(x, y, t)$ of problem (22)-(26).

Lemma 6 ([14]). If $a \geq b \geq 0, x \geq 0, \tau \in \mathbb{R}$, then

(1) $|\cosh (a+i \sigma b)| \geq c e^{a}, 0<c=\frac{\sqrt{1-2 e^{-\pi / 2}}}{2}<1$;

(2) $|\cosh (x(a+i \sigma b))| \leq e^{x a}$.

Theorem 3. Suppose problem (22)-(26) has a unique solution, let $u(x, y, t)$ given by $(31)$ be the exact data at $0<x \leq 1$, and $u_{\delta}(x, y, t)$ be the simplified Tikhonov regularized approximation to $u(x, y, t)$. Besides, assume $\varphi_{\delta}$ satisfy (28) and

$$
\|\psi\|_{p} \leq E, p \geq 0
$$

where $\|\cdot\|_{p}$ denotes the norm in Sobolev space $H^{p}\left(\mathbb{R}^{2}\right)$ defined by

$$
\|\psi\|_{p} \triangleq\left(\int_{\mathbb{R}^{2}}\left(1+\xi^{2}+\tau^{2}\right)^{p}|\hat{\psi}(\xi, \tau)|^{2} d \xi d \tau\right)^{\frac{1}{2}} .
$$

If $\alpha=\left(\frac{\delta}{E}\right)^{\gamma}, 1 \leq \gamma \leq 2$, then for $\forall 0<x \leq 1$,

$$
\begin{aligned}
& \left\|u_{\delta}(x, \cdot, \cdot)-u(x, \cdot, \cdot)\right\| \\
\leq & O\left(\delta^{(1-\gamma / 2) x}+\delta^{\gamma-(1+x) / 2} \cdot\left(\ln \frac{E}{\delta}\right)^{-(x+1) p / 2}+\left(\ln \frac{E}{\delta}\right)^{-p} \delta^{\gamma(1-x) / 2}\right) .
\end{aligned}
$$

Proof. Since

$$
\begin{aligned}
\left|\hat{u}_{\delta}-\hat{u}\right|^{2} & =\left|\hat{\varphi} \cosh (x \theta)-\hat{\varphi}_{\delta} \cdot \frac{\cosh (x \theta)}{1+\alpha|\cosh \theta|^{2}}\right|^{2} \\
& =\left|\frac{\left(\hat{\varphi}-\hat{\varphi}_{\delta}\right) \cosh (x \theta)+\alpha \hat{\varphi} \cosh (x \theta) \cdot|\cosh \theta|^{2}}{1+\alpha|\cosh \theta|^{2}}\right|^{2} \\
& \leq 2 \frac{\left|\hat{\varphi}-\hat{\varphi}_{\delta}\right|^{2} \cdot|\cosh (x \theta)|^{2}}{\left[1+\alpha|\cosh \theta|^{2}\right]^{2}}+2 \alpha^{2} \frac{|\hat{\varphi} \cosh \theta|^{2} \cdot|\cosh \theta|^{2} \cdot|\cosh (x \theta)|^{2}}{\left[1+\alpha|\cosh \theta|^{2}\right]^{2}} \\
& =2 \frac{\left|\hat{\varphi}-\hat{\varphi}_{\delta}\right|^{2} \cdot|\cosh (x \theta)|^{2}}{\left[1+\alpha|\cosh \theta|^{2}\right]^{2}}+2 \alpha^{2} \frac{|\hat{\psi}|^{2} \cdot|\cosh \theta|^{2} \cdot|\cosh (x \theta)|^{2}}{\left[1+\alpha|\cosh \theta|^{2}\right]^{2}} \\
& \leq 2\left|\hat{\varphi}-\hat{\varphi}_{\delta}\right|^{2} \cdot I_{1}+2 \alpha^{2}|\hat{\psi}|^{2} \cdot I_{2},
\end{aligned}
$$

where

$$
I_{1}=\frac{|\cosh (x \theta)|^{2}}{\left[1+\alpha|\cosh \theta|^{2}\right]^{2}}, \quad I_{2}=\frac{|\cosh \theta|^{2} \cdot|\cosh (x \theta)|^{2}}{\left[1+\alpha|\cosh \theta|^{2}\right]^{2}} .
$$

By Lemma 4 and Lemma 6 , we know that

$$
I_{1} \leq \frac{\left|e^{x a}\right|}{\left(1+\alpha c^{2} e^{2 a}\right)^{2}} \leq c^{-2} \frac{\left|e^{x a}\right|^{2}}{\left(1+\alpha e^{2 a}\right)^{2}} \leq c^{-2} \alpha^{-x} ;
$$




$$
I_{2} \leq \frac{\left|e^{a}\right|^{2} \cdot\left|e^{x a}\right|^{2}}{\left(1+\alpha c^{2} e^{2 a}\right)^{2}} \leq c^{-2} \frac{\left|e^{(1+x) a}\right|^{2}}{\left(1+\alpha e^{2 a}\right)^{2}} \leq c^{-2} \alpha^{-(1+x)} .
$$

Therefore,

$$
\begin{aligned}
\left\|\hat{u}_{\delta}(x, \xi, \tau)-\hat{u}(x, \xi, \tau)\right\|^{2} & \leq 2 \int_{\mathbb{R}^{2}}|\hat{\varphi}-\hat{\varphi} \delta|^{2} I_{1} d \xi+2 \alpha^{2} \int_{\mathbb{R}^{2}}|\hat{\psi}|^{2} I_{2} d \xi \\
& \leq 2 c^{-2} \delta^{2} \alpha^{-x}+2 \alpha^{2} \int_{\mathbb{R}^{2}}|\hat{\psi}|^{2} I_{2} d \xi
\end{aligned}
$$

Setting $m=\frac{1}{2} \ln \left(\frac{E}{\delta}\left(\ln \frac{E}{\delta}\right)^{-p}\right)$, define $\Omega_{m}=\left\{(\xi, \tau) \in \mathbb{R}^{2} \mid \frac{\xi^{2}}{m^{2}}+\frac{\tau^{2}}{4 m^{4}} \leq 1\right\}$.

It is easy to show that

$$
(\xi, \tau) \in \Omega_{m} \Leftrightarrow a \leq m
$$

and

$$
(\xi, \tau) \in \mathbb{R}^{2} \backslash \Omega_{m} \Rightarrow\left(1+\xi^{2}+\tau^{2}\right)^{-\frac{p}{2}} \leq m^{-p} .
$$

$$
\begin{aligned}
& 2 \alpha^{2} \int_{\mathbb{R}^{2}}|\hat{\psi}|^{2} I_{2} d \xi \\
\leq & 2 c^{-2} \alpha^{2} \int_{\Omega_{m}}|\hat{\psi}|^{2}\left(1+\xi^{2}+\tau^{2}\right)^{p}\left\{\frac{e^{(1+x) a}}{\left(1+\alpha e^{2 a}\right)\left(1+\xi^{2}+\tau^{2}\right)^{\frac{p}{2}}}\right\}^{2} \\
& +2 c^{-2} \alpha^{2} \int_{\mathbb{R}^{2} \backslash \Omega_{m}}|\hat{\psi}|^{2}\left(1+\xi^{2}+\tau^{2}\right)^{p}\left\{\frac{e^{(1+x) a}}{\left(1+\alpha e^{2 a}\right)\left(1+\xi^{2}+\tau^{2}\right)^{\frac{p}{2}}}\right\}^{2}
\end{aligned}
$$

$\triangleq J_{1}+J_{2}$.

Since $1+\xi^{2}+\tau^{2} \geq 1, a \leq m$ as $(\xi, \tau) \in \Omega_{m}$ and the function $f(s)=\frac{s}{1+\alpha^{2} s}$ is increasing for $s \geq 0$, then

$$
\begin{aligned}
J_{1} & \leq 2 c^{-2} \alpha^{2} E^{2} \sup _{0 \leq a \leq m}\left(\frac{e^{(1+x) a}}{1+\alpha e^{2 a}}\right)^{2} \\
& \leq 2 \alpha^{2} E^{2} c^{-2}\left(\frac{e^{(1+x) m}}{1+\alpha e^{2 m}}\right)^{2} \\
& \leq 2 E^{2} c^{-2} \alpha^{2} e^{2(1+x) m} \\
& =2 E^{2} c^{-2} \alpha^{2}\left(\frac{E}{\delta}\left(\ln \frac{E}{\delta}\right)^{-p}\right)^{1+x} \\
& =C_{1} \alpha^{2} \delta^{-(1+x)} \cdot\left(\ln \frac{E}{\delta}\right)^{-(1+x) p} .
\end{aligned}
$$

And

$$
\begin{aligned}
J_{2} & \leq 2 c^{-2} \alpha^{2} E^{2} \sup _{\mathbb{R}^{2} \backslash \Omega_{m}}\left\{\frac{e^{(1+x) a}}{\left(1+\alpha e^{2 a}\right)\left(1+\xi^{2}+\tau^{2}\right)^{\frac{p}{2}}}\right\}^{2} \\
& \leq 2 c^{-2} E^{2} m^{-2 p} \alpha^{1-x}=2^{2 p+1} c^{-2} E^{2}\left[\ln \left(\frac{E}{\delta}\left(\ln \frac{E}{\delta}\right)^{-p}\right)\right]^{-2 p} \alpha^{1-x} \\
& =2^{2 p+1} c^{-2} E^{2}\left(\ln \frac{E}{\delta}-p \ln \left(\ln \frac{E}{\delta}\right)\right)^{-2 p} \alpha^{1-x} \leq C_{2}\left(\ln \frac{E}{\delta}\right)^{-2 p} \alpha^{1-x} .
\end{aligned}
$$


Inserting (39)-(41) into (38), we get

$$
\begin{aligned}
& \left\|\hat{u}_{\delta}(x, \xi, \tau)-\hat{u}(x, \xi, \tau)\right\|^{2} \\
\leq & O\left(\delta^{2} \alpha^{-x}+\left(\alpha^{2} \delta^{-(1+x)} \cdot\left(\ln \frac{E}{\delta}\right)^{-(1+x) p}+\left(\ln \frac{E}{\delta}\right)^{-2 p} \alpha^{1-x}\right) .\right.
\end{aligned}
$$

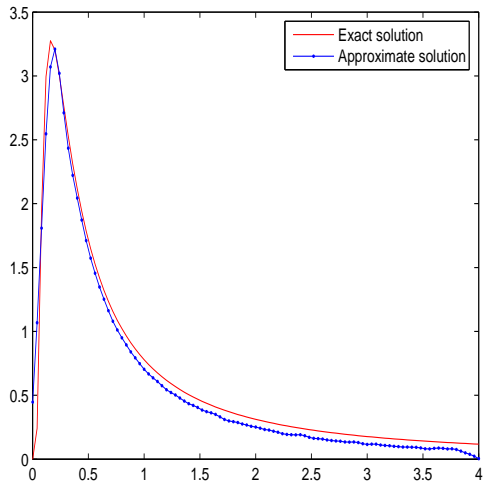

Figure 2. $x=0, p=$ $\frac{1}{3}, E=\|f\|_{\frac{1}{3}} \leq 3, \delta=$ 0.001 .

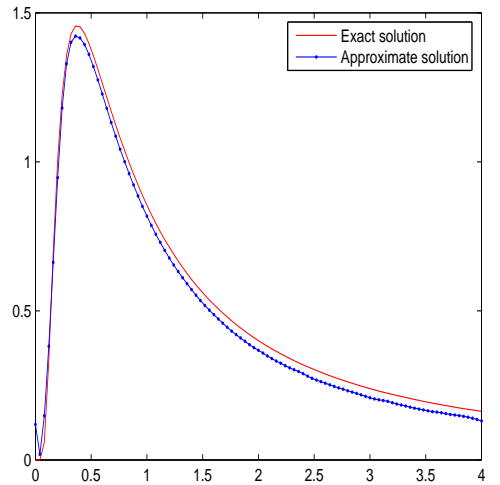

Figure $4 . x=0.5$, $p=\frac{1}{3}, E=\|f\|_{\frac{1}{3}} \leq 3$, $\delta=0.001$.

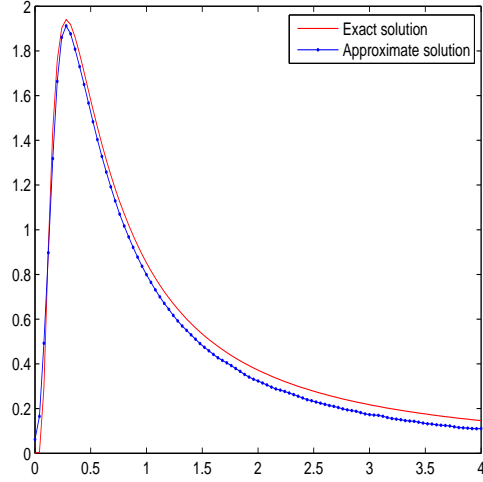

FiguRE $3 . x=0.3$, $p=\frac{1}{3}, E=\|f\|_{\frac{1}{3}} \leq 3$, $\delta=0.001$.

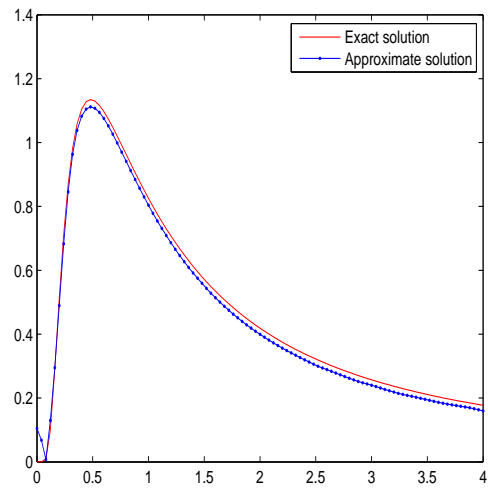

FiguRE 5. $x=0.7$, $p=\frac{1}{3}, E=\|f\|_{\frac{1}{3}} \leq 3$, $\delta=0.001$. 

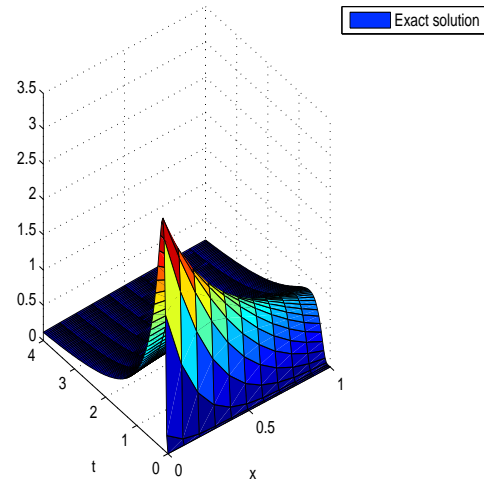

Tikhonve Regularization

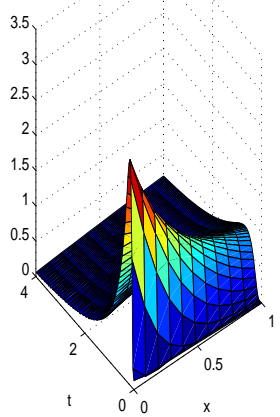

FigURE 6. $x=0, p=\frac{1}{3}, E=\|f\|_{\frac{1}{3}} \leq 3, \delta=0.001$.
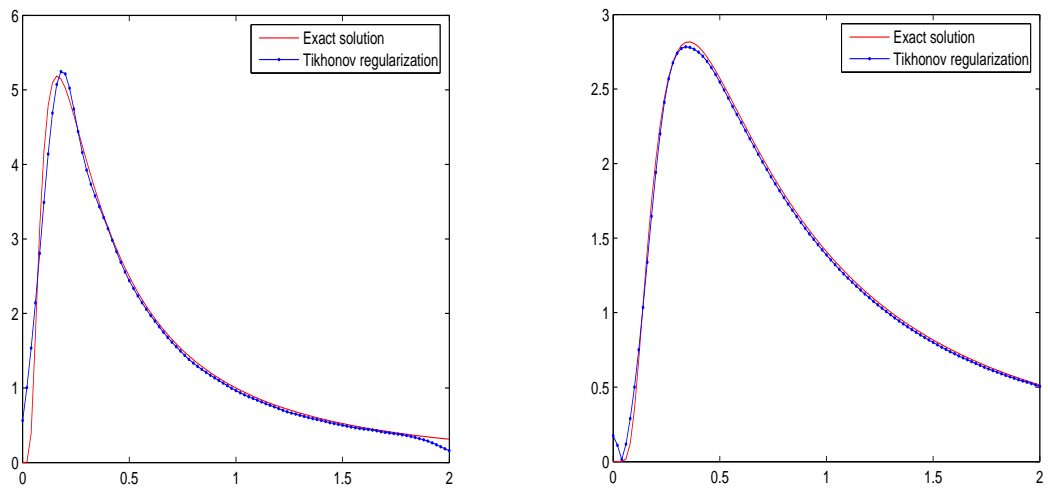

Figure 7. $x=0, p=$ $\frac{1}{3}, E=\|f\|_{\frac{1}{3}} \leq 3, \delta=$ 0.001 .

Figure $8 . x=0.5$, $p=\frac{1}{3}, E=\|f\|_{\frac{1}{3}} \leq 3$, $\delta=0.001$.

\section{Numerical experiments}

Example 1. It is easy to verify that

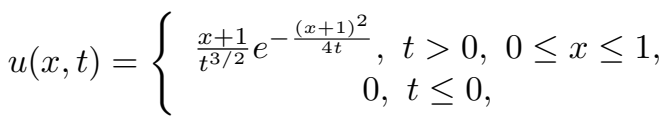




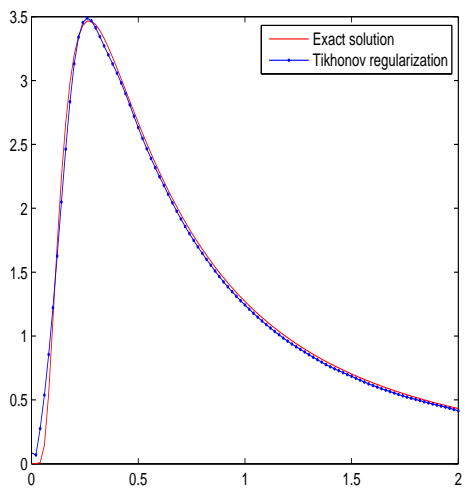

Figure $9 . x=0.3$, $p=\frac{1}{3}, E=\|f\|_{\frac{1}{3}} \leq 3$, $\delta=0.001$.

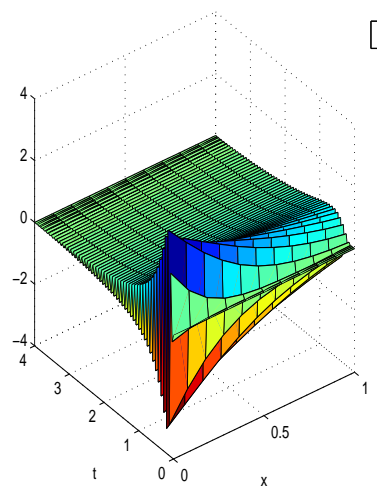

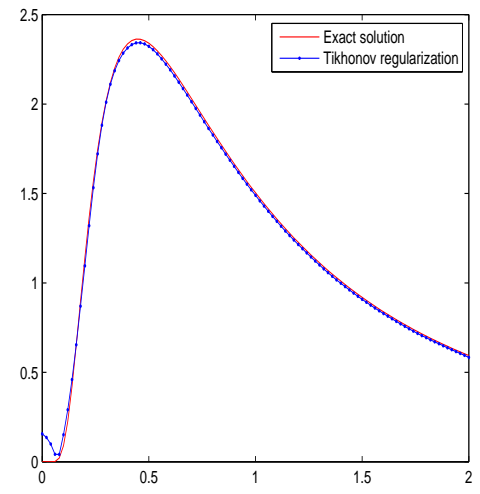

FiguRE 10. $x=0.7$, $p=\frac{1}{3}, E=\|f\|_{\frac{1}{3}} \leq 3$, $\delta=0.001$.

Figure 11. $x=0, p=\frac{1}{3}, E=\|f\|_{\frac{1}{3}} \leq 3, \delta=0.001$.

is the exact solution of problem (1)-(3) with $a(x)=1, b(x)=c(x)=0$ and with data

$$
\begin{aligned}
& g(t)=\left\{\begin{array}{c}
\frac{2}{t^{3 / 2}} e^{-\frac{1}{t}}, t>0, \quad g(t) \in L^{2}(\mathbb{R}) \\
0, t \leq 0,
\end{array}\right. \\
& f(t)=\left\{\begin{array}{c}
\frac{1}{t^{3 / 2}} e^{-\frac{1}{4 t}}, t>0, \quad f(t) \in H^{p}(\mathbb{R}), p \geq 0 . \\
0, t \leq 0,
\end{array}\right.
\end{aligned}
$$




\section{Example 2.}

$$
u(x, t)=\left\{\begin{array}{c}
\frac{x+1}{t^{3 / 2}} e^{-\frac{(x+1-t)^{2}}{4 t}}, t>0,0 \leq x \leq 1, \\
0, t \leq 0,
\end{array}\right.
$$

is the exact solution of (1)-(3) with $a(x)=1, b(x)=-1, c(x)=0$,

$$
\begin{aligned}
& g(t)=\left\{\begin{array}{c}
\frac{2}{t^{3 / 2}} e^{-\frac{(2-t)^{2}}{4 t}}, t>0, \quad g(t) \in L^{2}(\mathbb{R}) ; \\
0, t \leq 0,
\end{array}\right. \\
& f(t)=\left\{\begin{array}{c}
\frac{1}{t^{3 / 2}} e^{-\frac{(1-t)^{2}}{4 t}}, t>0, \quad f(t) \in H^{p}(\mathbb{R}), \quad p \geq 0 . \\
0, t \leq 0,
\end{array}\right.
\end{aligned}
$$

\section{References}

[1] L. Eldén, Numerical solution of the sideways heat equation by difference approximation in time, Inverse Problems 11 (1995), no. 4, 913-923.

[2] L. Eldén, F. Berntsson, and T. Regińska, Wavelet and Fourier methods for solving the sideways heat equation, SIAM J. Sci. Comput. 21 (2000), no. 6, 2187-2205.

[3] H. W. Engl, Regularization of Inverse Problem, Kluwer Academic Publishers, Bostom, 2000 .

[4] C. L. Fu, Simplified Tikhonov and Fourier regularization methods on a general sideways parabolic equation, J. Comput. Appl. Math. 167 (2004), no. 2, 449-463.

[5] L. Guo and D. A. Murio, A mollified space-marching finite-difference algorithm for the two-dimensional inverse heat conduction problem with slab symmetry, Inverse Problems 7 (1991), no. 2, 247-259.

[6] D. N. Hào, A mollification method for ill-posed problems, Numer. Math. 68 (1994), no. 4, 469-506.

[7] D. N. Hào and H. J. Reinhardt, On a sideways parabolic equation, Inverse Problems 13 (1997), no. 2, 297-309.

[8] D. N. Hào, H. J. Reinhardt, and A. Schneider, Numerical solution to a sideways parabolic equation, Internat. J. Numer. Methods Engrg. 50 (2001), no. 5, 1253-1267.

[9] Z. H. Liu, Browder-Tikhonov regularization of non-coercive evolution hemivariational inequalities, Inverse Problems 21 (2005), no. 1, 13-20.

[10] Z. H. Liu, J. Li, and Z. W. Li, Regularization method with two parameters for nonlinear ill-posed problems, Sci. China Ser. A 51 (2008), no. 1, 70-78.

[11] L. Liu and D. A. Murio, Numerical experiments in 2-D IHCP on bounded domains. I. The "interior" cube problem, Comput. Math. Appl. 31 (1996), no. 1, 15-32.

[12] D. A. Murio, The Mollification Method and the Numerical Solution of Ill-Posed Problems, A Wiley-Interscience Publication. John Wiley \& Sons, Inc., New York, 1993.

[13] M. T. Nair and U. Tautenhahn, Lavrentiev regularization for linear ill-posed problems under general source conditions, Z. Anal. Anwendungen 23 (2004), no. 1, 167-185.

[14] Z. Qian and C. L. Fu, Regularization strategies for a two-dimensional inverse heat conduction problem, Inverse Problems 23 (2007), no. 3, 1053-1068.

[15] T. Regińska and L. Eldén, Stability and convergence of the wavelet-Galerkin method for the sideways heat equation, J. Inverse Ill-Posed Probl. 8 (2000), no. 1, 31-49.

[16] M. Tadi, An iterative method for the solution of ill-posed parabolic systems, Appl. Math. Comput. 201 (2008), no. 1-2, 843-851.

[17] A. N. Tikhonov and V. Y. Arsenin, Solutions of Ill-Posed Problems, John Wiley \& Sons, New York-Toronto, Ont.-London, 1977.

[18] J. S. Tu and J. V. Beck, Solution of inverse heat conduction problems using a combined function specification and regularization method and a direct finite element solver, 
Proc. 2nd Ann. Inverse Problems in Engineering Seminar ed J. V. Beck (East Lansing, MI:College of Engineering, Michigan State University), (1989), pp. 1-25.

LI JING

School of Mathematics and Computational Science

Changsha University of Science and Technology

Changsha, Hunan 410075, P. R. China

E-mail address: lijingnew@126.com

WANG FANG

School of Mathematics and Computational Science

Changsha University of Science and Technology

Changsha, Hunan 410075, P. R. China

E-mail address: wangfang811209@tom.com 